\title{
Patient perception of scoliosis correction surgery on Instagram
}

\author{
Nitin Agarwal, MD, Amaan Rahman, Rachel Jacobs, MD, Tavis Taylor, BA, Nallammai Muthiah, BS, \\ Nima Alan, MD, Alp Ozpinar, MD, Daryl Fields, MD, and David Kojo Hamilton, MD
}

Department of Neurological Surgery, University of Pittsburgh Medical Center, Pittsburgh, Pennsylvania

\begin{abstract}
OBJECTIVE Patient feedback surveys provide important insight into patient outcomes, satisfaction, and perioperative needs. Recent critiques have questioned provider-initiated surveys and their capacity to accurately gauge patient perspectives due to intrinsic biases created by question framing. In this study, the authors sought to evaluate providerindependent, patient-controlled social media Instagram posts in order to better understand the patient experience following scoliosis correction surgery.

METHODS Twitter and Instagram were queried for posts with two tagged indicators, \#scoliosissurgery or @scoliosis surgery, resulting in no relevant Twitter posts and 25,000 Instagram posts. Of the initial search, 24,500 Instagram posts that did not directly involve the patient's own experience were eliminated. Posts were analyzed and coded for the following criteria: the gender of the patient, preoperative or postoperative timing discussed in the post, and classified themes related to the patient's experiences with scoliosis correction surgery.
\end{abstract}

RESULTS Females made $87.6 \%$ of the Instagram posts about their experience following scoliosis correction surgery. The initial postoperative stage of surgery was mentioned in $7.6 \%$ of Instagram posts. The most common theme on Instagram involved offering or seeking online support from other patients, which constituted $85.2 \%$ of all posts. Other common themes included concern about the surgical scar (31.8\%), discussing the results of treatment (28.8\%), and relief regarding results $(21.2 \%)$.

CONCLUSIONS Social media provided a platform to analyze unprompted feedback from patients. Patients were most concerned with their scoliosis correction surgery in the period of time 2 weeks or more after surgery. Themes that were most commonly found on Instagram posts were offering or seeking online support from other patients and concern about the surgical scar. Patient-controlled social media platforms, like Instagram, may provide a useful mechanism for healthcare providers to understand the patient experience following scoliosis correction surgery. Such platforms may help in evaluating postoperative satisfaction and improving postoperative quality of care.

https://thejns.org/doi/abs/10.3171/2021.8.FOCUS201015

KEYWORDS scoliosis; social media; spine; Instagram; surgery

$\mathrm{P}$ ATIENT feedback is a valuable resource for surgeons to assess patient satisfaction with surgical outcomes and improve postoperative quality of care. Traditionally, patient feedback has been measured through clinical surveys in medical settings or at clinic visits. Surveys provided in such settings are subject to the biases of social desirability and time lag..$^{1-3}$ Patients may express more positive feedback in front of their surgeon or on clinical surveys to maintain positive social relationships. Furthermore, many neurosurgical clinics are quick and directed, so patients may not have the time to voice emotional or aesthetic concerns. Surveys in the postoperative clinical setting also induce an artificial, fixed time lag between treatment and outcome measurement such that patient responses are not necessarily representative of their full postoperative experience. ${ }^{3,4}$ It is also not surprising that patients who respond to healthcare surveys likely do not accurately reflect the entire population of postoperative patients. ${ }^{3,5,6}$ Not only do surveys in clinical settings pose a selection bias against patients of lower socioeconomic status and those of an ethnic minority, ${ }^{6}$ but they are also prone to social desirability bias ${ }^{2,6}$ and loss of nuanced perception of patient experience due to the artificial and fixed time lag between surgery and clinical follow-up. ${ }^{3}$

Over the last few decades, social media has become a powerful tool for disseminating information between the 
TABLE 1. Coded themes of Instagram posts

\begin{tabular}{|c|c|}
\hline Theme & Criteria \\
\hline Scheduling or referencing upcoming treatment & If post relates to a procedure or appointment that has yet to come. \\
\hline Discussing results of treatment & Anything explicitly mentioning the results of treatment or nonsurgical procedures. \\
\hline $\begin{array}{l}\text { Discussing ongoing treatment experience } \\
\text { (treatment experience update) }\end{array}$ & $\begin{array}{l}\text { Patient is clearly going through treatment at the time of the post, or providing new updates on } \\
\text { their treatment journey. }\end{array}$ \\
\hline Use of humor & Clear sarcasm, use of laughing emoji, or making a joke at the expense of someone or self. \\
\hline Advising others to get treatment & $\begin{array}{l}\text { Any type of message, hashtag, or phrase encouraging others to somehow monitor their } \\
\text { scoliosis-related health. }\end{array}$ \\
\hline Mentioning a specific health provider & $\begin{array}{l}\text { If post is posted by, or mentions in any way, shape, or form, a physician, healthcare profes- } \\
\text { sional, hospital, or overall service that will aid, is aiding, or has aided the patient. }\end{array}$ \\
\hline Process of waiting & Specific frustration w/ the lead-up to a diagnosis, procedure, or surgery. \\
\hline Prepping for surgery & A patient update that is w/in 2 days prior to surgery \& specifically mentions the impending surgery. \\
\hline Relief regarding results & $\begin{array}{l}\text { Feeling of reassurance \& relaxation after spinal fusion surgery; no time frame for this charac- } \\
\text { teristic, it only needs to be after the surgery. }\end{array}$ \\
\hline $\begin{array}{l}\text { General apprehension regarding undergoing } \\
\text { treatment }\end{array}$ & $\begin{array}{l}\text { General worry or stress during the time frame in which a patient is considered inpatient. Worry } \\
\text { is separated from frustration in this analysis. Worry coincides w/ stress w/ regard to a hypo- } \\
\text { thetical scenario. Frustration has to do w/ annoyance at the current situation. This is what } \\
\text { makes this characteristic separate from "process of waiting." }\end{array}$ \\
\hline Treatment costs & Any positive or negative mention of the financial impact of treatment. \\
\hline Dissatisfaction w/ results & Negative feelings specifically \& explicitly toward the results of the surgery. \\
\hline Postop pain & Specifically discussing patient pain after surgery. \\
\hline Preop pain & Specifically discussing patient pain prior to an upcoming surgery. \\
\hline Concern about surgical scar & Picture of scar or written/verbal mention of scar. \\
\hline Offering or seeking online supp & Giving a positive or generally encouraging message \&/or asking f \\
\hline
\end{tabular}

general population and healthcare providers. Today, $72 \%$ of all adult Americans use social media. ${ }^{7}$ Social media influences patient beliefs, choices, and actions in healthcare decision-making. ${ }^{4,5,8}$ As it is relatively accessible, the internet has become a more prevalent source of healthcare information. This information can be either formal (i.e., messaging systems through healthcare-provided media) or informal (e.g., general social media and blogs). To this end, social media allows patients to exchange information through a healthcare provider-independent medium.

Recent studies have also utilized social media to understand patient experience with regard to specific procedures or medical subspecialties such as Gamma Knife stereotactic radiosurgery ${ }^{9}$ and neurosurgery. ${ }^{10}$ Collectively, these studies have found social media useful for attaining accurate patient feedback. Similarly, in this study, we aimed to understand what themes and characteristics were most relevant to patients undergoing scoliosis correction surgery.

\section{Methods}

Twitter and Instagram were searched for several hashtags pertaining to deformity correction surgery to identify posts that elucidated the patient experience of scoliosis correction. The searched hashtags were: \#deformitysurgery (Instagram); \#scoliosistreatment (Instagram); \#scoliosistreatment (Twitter); \#scoliosissurgery (Twitter); \#scoliosissurgery (Instagram); \#scoliosis\#surgery (Twitter); \#scoliosis\#surgery
(Instagram); \#scoliosis\#spinalfusion (Instagram); \#scoliosis spinalfusion (Instagram); \#scoliosis\#spinalfusion (Twitter); and \#scoliosisspinalfusion (Twitter).

The Twitter search resulted in no relevant posts; however, on Instagram, the hashtag \#scoliosissurgery was the most encompassing, resulting in 25,000 posts related to spine deformity correction. Of the 25,000 posts with the hashtag \#scoliosissurgery, 500 posts were selected. Posts were included only if they directly discussed patient experience with scoliosis correction surgery. Posts were not excluded based on the identity of the poster; therefore, patients, medical professionals, foundation members, parents, and guardians were all potential sources for the qualitative data in this study. Posts that did not directly discuss patient experience were excluded.

Posts were subsequently categorized into themes, as shown in Table 1. Themes were not mutually exclusive. While each condition had a unique set of traits, it was possible for the content of a post to satisfy multiple characteristics or themes. For example, when patients posted after surgery and also referenced their preoperative experiences, this satisfied both preoperative and postoperative characteristics.

Regarding the postoperative time dichotomization in this study, any time within 2 weeks of the surgery was considered initial postoperative, and any subsequent time was considered 2 weeks or more after surgery. Surgery that took place after the initial scoliosis correction surgery was considered repeat surgery. When posts referred to 
TABLE 2. Characteristics of the 500 included Instagram posts

\begin{tabular}{cc}
\hline Characteristic & Instagram Frequency \\
\hline Gender* & \\
\hline M & $43(8.6)$ \\
\hline F & $438(87.6)$ \\
\hline Time periodt & $63(12.6)$ \\
\hline Preop & $38(7.6)$ \\
\hline Initial postop & $437(87.4)$ \\
\hline$\geq 2$ wks postop & $12(2.4)$ \\
\hline Repeat surgery
\end{tabular}

Values represent the number of posts (\%).

${ }^{*}$ A minority of posts lacked identifying information and were excluded from these frequency totals.

† Overlap occurred due to certain posts satisfying multiple characteristics, thus constituting a percentage greater than $100 \%$.

multiple perioperative time periods, they were coded under each relevant time point. Thus, the frequency of these data tallied to $>100 \%$.

\section{Results}

Among the posts included in this analysis, $87.6 \%$ were from females $(8.6 \%$ were from males, and gender was unknown in $4.0 \%$ ). Of the 500 coded Instagram posts, $12.6 \%$ mentioned the preoperative period, $87.4 \%$ mentioned the 2 weeks or more after surgery, $7.6 \%$ mentioned the initial postoperative period, and $2.4 \%$ mentioned a repeat surgery period (Table 2 )

The frequencies of the major Instagram post themes are demonstrated in Table 3. Overall, $85.4 \%$ of posts involved offering or seeking online support from other patients. Other common themes included concern about the surgical scar (31.8\%; Fig. 1), discussing the results of treatment $(28.8 \%)$, relief regarding results $(21.2 \%)$, and postoperative pain $(20.8 \%)$

Certain themes were often mentioned together. In total, the process of waiting was mentioned in $11.4 \%$ of posts, while prepping for surgery was mentioned in $9.8 \%$ of posts. Of the posts regarding prepping for surgery, $91.8 \%$ also mentioned the process of waiting. In addition, offering or seeking online support from other patients was conveyed in $79 \%$ of posts mentioning postoperative pain. Of posts that discussed the results of treatment, $76.5 \%$ also mentioned offering or seeking online support from other patients.

\section{Discussion}

The use of social media can serve as an effective tool for gathering and interpreting patient feedback for previous medical procedures. ${ }^{11-13}$ This study identified key characteristics expressed by patients on Instagram in relation to their experiences with scoliosis correction surgery.

\section{Gender-Related Data}

The majority of posts $(87.6 \%)$ were made by females. This is not necessarily surprising, as approximately $78 \%$
TABLE 3. Frequency of themes in Instagram posts regarding the patient experience

\begin{tabular}{lc}
\hline \multicolumn{1}{c}{ Theme } & No. of Posts (\%) \\
\hline $\begin{array}{l}\text { Offering or seeking online support from other } \\
\text { patients }\end{array}$ & $427(85.4)$ \\
\hline Concerns about surgical scar & $159(31.8)$ \\
\hline Discussing results of treatment & $144(28.8)$ \\
\hline Relief regarding results & $106(21.2)$ \\
\hline Postop pain & $104(20.8)$ \\
\hline Preop pain & $65(13.0)$ \\
\hline Process of waiting & $57(11.4)$ \\
\hline Scheduling or referencing upcoming treatment & $54(10.8)$ \\
\hline Prepping for surgery & $49(9.8)$ \\
\hline Mentioning a specific health provider & $44(8.8)$ \\
\hline Use of humor & $40(8.0)$ \\
\hline $\begin{array}{l}\text { Discussing ongoing treatment experience } \\
\text { (treatment experience update) }\end{array}$ & $38(7.6)$ \\
\hline $\begin{array}{l}\text { General apprehension regarding undergoing } \\
\text { treatment }\end{array}$ & $24(4.8)$ \\
\hline Dissatisfaction w/ results & $22(4.4)$ \\
\hline Advising others to get treatment & $16(3.2)$ \\
\hline Treatment costs & $1(0.2)$ \\
\hline Overapocurred &
\end{tabular}

Overlap occurred due to certain posts satisfying multiple themes, thus constituting a percentage greater than $100 \%$.

of females and $65 \%$ of males use social media. ${ }^{7}$ About $4 \%$ of posts were created by people whose gender was not explicitly discernible. Despite that the majority of posts were created by females, both males and females primarily used Instagram as a source of social support for scoliosis correction surgery ( $84.5 \%$ of females and $86 \%$ of males). This is almost $30 \%$ greater than the percentage of female Instagram users (58\%), ${ }^{14,15}$ suggesting that other factors likely contributed to our sample's female preponderance. A likely explanation is the higher prevalence of idiopathic scoliosis in females compared with males. ${ }^{11}$

\section{Operative Time Period}

Among posts, $87.4 \%$ met criteria for 2 weeks or more after surgery. Yet, as most posts mentioned the 2-week period within and after the initial postoperative wound check, this time frame should be regarded as important to most patients. Even acting independently without prompting, patients felt it important to discuss their experience on Instagram during this time. Therefore, the patient-physician relationship can be important to the patient's postoperative experience. Coincidentally, this is also the period in which patients have been found to report the most pain. ${ }^{13}$ Strong patient support during the 2 weeks or more postoperatively can reinforce provider engagement in care and improve patient satisfaction.

\section{Satisfaction With Results and Cost}

Some may argue that social media tends to filter out negative content. In our cohort, patients posted negative 


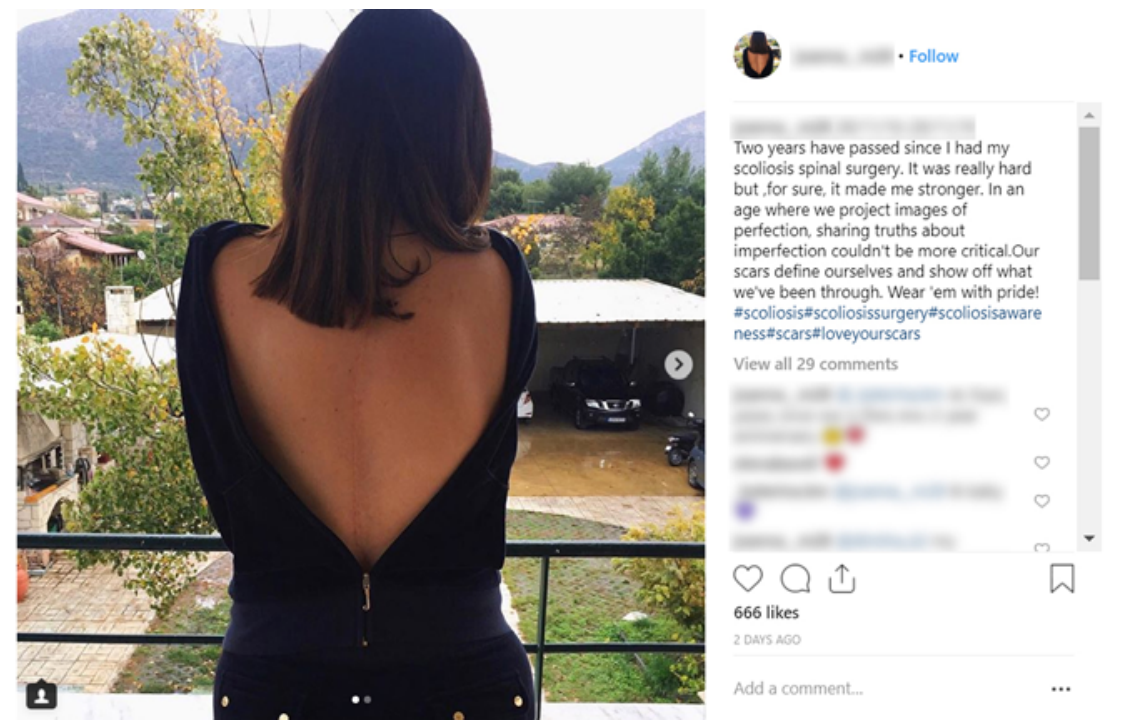

FIG. 1. Instagram post exemplifying the theme related to concern about the surgical scar.

content without expressing explicit dissatisfaction with results. Only $4.4 \%$ of patients explicitly mentioned dissatisfaction with the surgery results. At the same time, $20.8 \%$ of posts mentioned postoperative pain and $31.8 \%$ mentioned concern about the surgical scar. In fact, $27 \%$ of adolescent patients who underwent scoliosis correction surgery have reportedly not been satisfied with the appearance of their scar following the operation. ${ }^{10}$

Only 1 reviewed post out of 500 mentioned the cost of treatment as an issue. Social media users come from a variety of socioeconomic backgrounds, ${ }^{16}$ and a myriad of reasons may underlie the lack of discourse regarding cost. It is possible that the lack of posts with fiscal content may suggest insurance companies are providing appropriate treatment authorizations. Still, the issue of prior authorization remains a source of great contention and may be under appreciated. According to the Journal of the American College of Cardiology, $78 \%$ of physicians reported treatment abandonment by patients due to prior authorization delays; $64 \%$ of these same respondents reported having to wait at least 1 business delay for insurers to provide preapproval for a procedure. ${ }^{17}$

Social media may provide an additional source for healthcare providers to understand how patients feel in the perioperative time period. Unprompted feedback online lacks framing biases often implicit in healthcare-provided surveys. The difficulty of eliminating intrinsic survey bias is also confounded by patient response variations as patient survey responders may represent a small subpopulation of operative patients. Lasek et al. found that patients who opted not to respond tended to have both longer hospital stays and nonsurgical stays. ${ }^{18}$ Mazor et al. found that the majority of patients who did respond tended to be highly passionate about their satisfaction or dissatisfaction. ${ }^{2}$ Consequently, healthcare-provided surveys may not accurately reflect the true beliefs, opinions, and feelings of the patients. Due to these findings, the importance of social media as a tool for deciphering the unframed feed- back of patients has heightened vastly. Recognizing the ability of social media users to spread health-related opinions and feedback to wide audiences, it is important that healthcare professionals understand current online content as it pertains to their patients.

\section{Limitations}

It is important to note that the study has several limitations. First and foremost, social media is inherently filtered. This poses a selection bias-not all patients who underwent scoliosis surgery will post on Instagram about their recovery. It is difficult to ascertain the true generalizability of our results. Another limitation is the inability to discern the true time period at which the post occurred due to the nature of social media. As described in Methods, the time frames were coded based on information from the posts. Moreover, several posts addressed multiple perioperative time periods. Understanding at which specific perioperative time point a post was created may provide a better picture of patient feedback and consequently where to concentrate quality improvement strategies. Finally, the age of posters was unknown. Additional research is required to determine differences in content (i.e., appearance of surgical scars) among age groups.

\section{Conclusions}

Social media provides a platform to analyze unprompted feedback from patients. Patients were most concerned with their scoliosis correction surgery in the period 2 weeks or more after surgery. The themes most commonly found on Instagram posts were offering or seeking online support from other patients and concern about the surgical scar. Patient-controlled social media platforms, like Instagram, may provide a useful mechanism for healthcare providers to understand the patient experience following scoliosis correction surgery. Such platforms may help in 
evaluating postoperative satisfaction and improving postoperative quality of care.

\section{References}

1. Hekkert KD, Cihangir S, Kleefstra SM, van den Berg B, Kool RB. Patient satisfaction revisited: a multilevel approach. Soc Sci Med. 2009;69(1):68-75.

2. Mazor KM, Clauser BE, Field T, Yood RA, Gurwitz JH. A demonstration of the impact of response bias on the results of patient satisfaction surveys. Health Serv Res. 2002;37(5): 1403-1417.

3. Verhoef LM, Van de Belt TH, Engelen LJ, Schoonhoven L, Kool RB. Social media and rating sites as tools to understanding quality of care: a scoping review. J Med Internet Res. 2014;16(2):e56.

4. Seltzer EK, Horst-Martz E, Lu M, Merchant RM. Public sentiment and discourse about Zika virus on Instagram. Public Health. 2017;150:170-175.

5. Chandrasekaran N, Gressick K, Singh V, et al. The utility of social media in providing information on Zika virus. Cureus. 2017;9(10):e1792.

6. Lovaglio PG. Benchmarking strategies for measuring the quality of healthcare: problems and prospects. ScientificWorldJournal. 2012;2012:606154.

7. Pew Research Center. Social Media Fact Sheet. June 12, 2019. Accessed September 7, 2021. https://www.pewresearch. org/internet/fact-sheet/social-media/

8. Waring ME, Jake-Schoffman DE, Holovatska MM, Mejia C, Williams JC, Pagoto SL. Social media and obesity in adults: a review of recent research and future directions. Curr Diab Rep. 2018;18(6):34.

9. Jacobs R, Prabhu AV, Monaco EA, Tonetti D, Agarwal N. Patient perception of Gamma Knife stereotactic radiosurgery through Twitter and Instagram. Interdiscip Neurosurg. 2018; 13:138-140.

10. Buchanan R, Birch JG, Morton AA, Browne RH. Do you see what I see? Looking at scoliosis surgical outcomes through orthopedists' eyes. Spine (Phila Pa 1976). 2003;28(24):27002705.

11. Roberts DW, Savage JW, Schwartz DG, et al. Male-female differences in Scoliosis Research Society-30 scores in adolescent idiopathic scoliosis. Spine (Phila Pa 1976). 2011; 36(1):E53-E59.

12. Antheunis ML, Tates K, Nieboer TE. Patients' and health professionals' use of social media in health care: motives, barriers and expectations. Patient Educ Couns. 2013;92(3): 426-431.
13. Connelly M, Fulmer RD, Prohaska J, et al. Predictors of postoperative pain trajectories in adolescent idiopathic scoliosis. Spine (Phila Pa 1976). 2014;39(3):E174-E181.

14. Vermeren I. Men vs. women: who is more active on social media? Brandwatch Bulletin. January 28, 2015. Accessed September 7, 2021. https://www.brandwatch.com/blog/menvs-women-active-social-media/

15. Lampos V, Aletras N, Geyti JK, Zou B, Cox IJ. Inferring the Socioeconomic Status of Social Media Users Based on Behaviour and Language. Springer; 2016.

16. Han X, Cao S, Shen Z, et al. Emergence of communities and diversity in social networks. Proc Natl Acad Sci U S A. 2017; 114(11):2887-2891.

17. Miller AP, Shor R, Waites T, Wilson BH. Prior authorization reform for better patient care. J Am Coll Cardiol. 2018; 71(17):1937-1939.

18. Lasek RJ, Barkley W, Harper DL, Rosenthal GE. An evaluation of the impact of nonresponse bias on patient satisfaction surveys. Med Care. 1997;35(6):646-652.

\section{Disclosures}

Dr. Agarwal: royalties from Thieme Medical Publishers.

\section{Author Contributions}

Conception and design: Agarwal, Jacobs. Acquisition of data: Agarwal, Rahman. Drafting the article: Agarwal, Rahman, Taylor. Critically revising the article: Agarwal, Jacobs, Muthiah, Alan, Ozpinar, Fields, Hamilton. Reviewed submitted version of manuscript: Agarwal. Approved the final version of the manuscript on behalf of all authors: Agarwal. Study supervision: Hamilton.

\section{Supplemental Information \\ Videos}

Video Abstract. https://vimeo.com/623493470.

\section{Correspondence}

Nitin Agarwal: University of Pittsburgh Medical Center, Pittsburgh, PA. agarwaln@upmc.edu. 